\title{
АНАЛИЗ ЭФФЕКТИВНОСТИ ЛЕЧЕНИЯ БОЛЬНЫХ АКРОМЕГАЛИЕЙ В ЕКАТЕРИНБУРГЕ
}

\author{
'Возжаева М.А., 'Сентюрина Л.Б., 'Кочергина М.В., ${ }^{1,2}$ Киселева Т.П.
}

'ГАУЗ СО «Городская клиническая больница № 40», амбулаторно- консультативное отделение эндокринологического иентра, Екатеринбург

2ФГБОУ ВО «Уральский государственный медицинский университет» Минздрава России, Екатеринбург

ЦЕЛЬ: провести анализ эффективности лечения диспансерных больных акромегалией в Городском эндокринологическом центре г. Екатеринбурга.

МАТЕРИАЛЫ И МЕТОДЫ: представлены данные 68 пациентов в возрасте от 22 до 85 лет: 50 женщин (74\%), 18 мужчин (26\%). Использовались общеклинические методы обследования, уровень ИРФ-1, исследование концентрации СТГ каждые 30 минут в течение 2-х часов при проведении орального глюкозотолерантного теста (ОГТТ) с 75 г. глюкозы, МРТ гипофиза с контрастированием.

PЕЗУЛЬТАТЫ: распространенность акромегалии в г. Екатеринбурге составляет 53,3 случая на 1 млн. Средний возраст пациентов - 43 года (мужчины - 37 лет, женщины - 46 лет). По размеру соматотропином: макроаденома гипофиза - 41 (60,3\%), микроаденома - 27 (39,7\%). У мужчин отмечается бо́льший размер аденом: 88,8\% (16 пациентов) - макроаденома, 11,2\% (2 пациента) - микроаденома. Среди женщин отмечается равномерное распределение: 50\% (25 пациенток) - макроаденома, 50\% (25 пациенток) - микроаденома. Диагностированные осложнения акромегалии: нарушения углеводного обмена, включая сахарный диабет - 57\%, узловой $306-53 \%$, артериальная гипертензия - 40\%. Активность акромегалии в стадии контроля - 25 пациентов (36,8\%), 43 пациента $(63,2 \%)$ - активная стадия заболевания. Нейрохирургическое лечение проведено 39 (57,3\%) пациентам: первичное - 27, вторичное - 12. Повторное оперативное вмешательство -6 больным. Полная ремиссия заболевания - 6 (16\%) случаев. 33 пациентам проведена вторичная медикаментозная и лучевая терапия. При хирургическом и комбинированном лечения контроль акромегалии достигнут у 13 (33,3\%) больных. Медикаментозная терапия (МT) - (аналоги соматостатина первой генерации, селективный агонист дофамина, а также антагонист рецепторов гормона роста) - проведена 48 (70\%) больным: первичная - 27 пациентов, вторичная 21 пациент. Маркеры эффективности терапии акромегалии - мониторинг уровня ИРФ-1, а также ИРФ-1 индекс. Медикаментозный контроль достигнут у 14 больных (29,2\%; при первичной МТ - 6, при вторичной - 8). Лучевая терапия (ЛТ) проведена 12 пациентам (3 - первичная, при наличии противопоказаний к хирургическому лечению; 9 - вторичная), ремиссия акромегалии достигнута у 4 (33,3\%) пациентов.

ВЫВОды: нейрохирургические методы лечения повышают эффективность лечения больных акромегалией, особенно с учетом размеров и распространения опухоли, а эффективность МТ сопряжена с развитием резистентности к ней, обусловленной морфологическими особенностями опухолей. Использование ЛТ следует проводить как дополнительный метод лечения при нерадикальном нейрохирургическом лечении и при резистентности к MT, в качестве первичного метода при наличии противопоказаний к нейрохирургическому лечению. 